\title{
Les Apprenants Yoroubaphones Du Français Face À La Lecture Du Texte En Français: Une Étude Phonostylistique
}

\author{
Adebayo, Lukman Adedoyin \\ Department of Mass Communication, Lead City University, Ibadan, Oyo State, Nigeria.
}

\begin{abstract}
Résumé : Beaucoup d'entre nous pensent que la lecture est un fait simple, un processus passif qui inclut la lecture des mots et la compréhension du sens de ces mots. La lecture n'est pas un processus simple comme nous le prenons, car elle demande une participation active de la part du lecteur.

Dans cet article, nous étudions le problème de la lecture chez les apprenants yoroubaphones du français au Nigéria. Nous sommes d'avis que l'articulation, l'intonation et le style jouent de rôles importants dans la lecture d'un texte écrit en français. À la fin de cette rencontre, nous avons proposé des techniques à prendre en compte pour rendre plus intéressant l'enseignement et l'apprentissage de la lecture en français au Nigéria en général et surtout chez les locuteurs d'origine yorouba.
\end{abstract}

Mot-clé : apprenants yoroubaphones, la lecture, phonostylistique, texte en français,

\section{Introduction}

Le français occupe une place importante dans la sphère internationale et c'est aussi une langue dominante employée comme moyen de communication par des organisations internationales dont le Nigéria est membre. Pour cette raison, les centres d'apprentissage et le désir pour son apprentissage augmentent. Étant donné que le français est une langue exogène pour les nigérians, son apprentissage et son enseignement entraînent des interférences linguistiques. Les chercheurs et les linguistes nigérians francisants aussi ont beaucoup travaillé pour résoudre le problème associé à l'apprentissage d'une langue étrangère comme le français et l'interférence n'échappe pas à leur regarde.

Dans cet article nous nous bornons à l'aspect phonostylistique de ce phénomène d'interférence linguistique. La phonostylistique s'intéresse à l'ensemble de tout ce qui a été ou est susceptible d'être oralisé et qui produit un effet par rapport au discours attendu. C'est la variabilité phonique, essentiellement humain, et celle de perception donnant une information supplémentaire. Comme nous savons que la langue n'est pas seulement la combinaison des sons pour former des mots ni celle des mots pour former des phrases, mais qu'il y a aussi des éléments prosodiques et stylistiques qui jouent des rôles importants. Ceci est d'autant vrai pour le français que Makouta-Mboukou (1973:65) écrit: "la langue française...ce n'est pas seulement l'exactitude structurale, mais aussi la bonne correction articulatoire" (cité par Jimoh (2007: 8) [1].

Le yorouba appartient à la famille des langues nigéro-congolaises. Selon les écoles linguistiques, la langue yorouba est une langue tonale mais le français est une langue accentué. Ces deux langues se manifestent sous deux formes à savoir l'oral et l'écrit. La forme est l'artifice que l'homme emploie pour désigner une certaine réalité. Autrement dit, c'est l'organisation conventionnelle des lettres à l'écrit et des sons à l'oral. La forme orale repose sur les phonèmes (les sons) de la langue tandis que la forme écrite ou graphique repose sur les graphèmes de la langue (les lettres).

\section{Définition de la lecture}

Beaucoup d'entre nous pensent que la lecture est un fait simple, un processus passif qui inclut la lecture des mots et la compréhension du sens de ces mots. La lecture n'est pas un processus simple comme nous le prenons, car elle demande une participation active de la part du lecteur. Tout d'abord, précisons que nous ne nous intéressons à la définition de la lecture que dans la mesure où elle correspond à la compréhension. Donc, comprendre un texte signifie construire sa signification.

D'après Falcoz (1987) [2], du point de vue didactique, la lecture est

La capacité de construire une signification à partir de ce qu'on perçoit, selon des modalités ou des stratégies dont le lecteur est maître et qu'il choisit à la fois en fonction des types de texte et de son projet de lecture.

Selon Braille (1980) [3]

La lecture est l'activité de compréhension d'une information écrite. Cette information en général est une représentation du langage sous forme de 
symboles identifiables par la vue ou par le toucher. (C'est nous qui soulignons).

D’après Smith (1997) [4]

Reading is a psycholinguistic guessing game. It involves an interaction between thought and language.

La lecture est un jeu de devinette psycholinguistique. Cela implique une interaction entre la langue et la pensée. (Notre traduction).

D'autre part Ogunola (2003) [5] définit la lecture comme

... la recherche d'une pensée qui existe dans l'esprit de l'auteur et qui se trouve en face du lecteur et que ce dernier doit traiter afin de la distiller pour en extraire la substance.

D’après Oloruntoba-Oju (1998) [6]

Reading involves a conscious activity of trying to get out the meaning of a given text in order to be able to appreciate the total import of the text.

La lecture est une activité délibérée qui consiste à faire sortir le message dans un texte donné pour pouvoir apprécier la signification du texte. (Notre traduction)

La lecture n'est pas tout simplement la perception et l'identification des éléments pertinents à la lecture mais elle demande des compétences et des techniques qui doivent se réunir pour qu'on parle d'un bon lecteur. Elle implique faire sortir ce qui est caché dans l'écrit. Les livres représentent traditionnellement l'écrit mais aujourd'hui, l'élève lit d'autres documents écrits tels que le logiciel de l'ordinateur, les informations sur l'écran de télévision et d'autres formes d'écrit.

\section{Phonostylistique: La définition}

L’Encyclopédie Hachette Multimédia (2003) [7] définit la phonostylistique comme Une étude des phénomènes ayant une valeur expressive (traduisant l'émotion du locuteur, son milieu socioculturel, son âge, son sexe, etc.) ou une valeur appellative (provoquant certains sentiments chez l'auditeur).

Le dictionnaire de la linguistique (1973: 377) [8] définit la phonostylistique comme :

Une partie de la phonologie qui étudie les éléments phoniques ayant dans le langage humain une fonction expressive (émotive) ou appellative (conative), mais non représentative (référentielle).

Bühler (1934) [9], dans son œuvre "Principes de phonologie" proposait de distinguer deux domaines de la phonostylistique: une stylistique phonologique et une stylistique phonétique. La première s'intéresse à l'étude des signes conventionnels, codes dans la langue tel que l'accent d'insistance en français, alors que la deuxième s'intéresse à l'étude des non-codés dans la langue tels que la toux, le bégaiement, etc.

Dans la phonostylistique on distingue la fonction phonologique qui permet de différencier entre deux mots tels 'saule' /sol/ (arbre ou arbuste qui croît dans les lieux frais et humides) et 'sol' /s l// (partie superficielle de la croûte terrestre) et entre deux phrases telles il pleut /2 $1 /$ (déclarative) et il pleut? $\quad / 2 \quad 4 /$ (interrogative). Ces exemples démontrent le mécanisme oppositif de la fonction phonologique: les mots se distinguent par l'opposition vocalique /o/ (mi-fermée), /J/ (mi-ourte) et les phrases par l'oppositient Intonative (courbe descendante versus courbe ascendante).

La deuxième fonction phonostylistique apporte au message oral une information supplémentaire. Par contraste de la fonction phonologique, la fonction phonostylistique ne se fond pas sur la notion d'une opposition linguistique pertinente. Par exemple "il pleut" peut transmettre en même temps de la colère, de la surprise, un accent régional, un effet emphatique, etc. Mais Ladefoged (1982:285) [10] est de l'avis que le locuteur manipule des faits prosodiques/suprasegmentaux de parole, telles que l'accentuation et l'intonation, qui ne sont des propriétés ni des consonnes ni des voyelles simples. De tels caractères de la parole, exploités dans la lecture d'un texte écrit peuvent, sans changer le message linguistique pour autant, influencer la réception du message par l'auditeur; à cet effet, il s'agit d'une question de style vocal. Le style vocal est défini comme une façon de 
parler, une manière de s'exprimer. Cette interprétation suggère, comme le remarque Fónagy (1983) [11] qu'une même phrase peut être dite de différentes façons, ce qui implique, de nouveau, la notion d'un choix.

En un mot, la fonction phonostylistique recouvre deux sous-fonctions principales: l'une "expressive" et l'autre "appellative". La fonction expressive caractérise le sujet parlant (son origine sociale, son âge, son sexe, sa culture, sa provenance géographique, etc.) tandis que la fonction appellative est destinée à déclencher certaines impressions chez l'auditeur. Il faut néanmoins considérer que la fonction expressive peut, elle aussi, évoquer certaines impressions ou émotions chez l'auditeur.

\section{Méthodologie}

Pour notre étude, nous avons enquêté 58 étudiants du Département de français du Collège Fédéral d'Éducation d'Abeokuta dans l'état d'Ogun. Ces étudiants sont composés de quarante filles et dix-huit garçons dont l'âge se situe entre dix-neuf et trente ans. Ils sont issus de familles yoroubaphones et vingt-cinq (22) parmi eux sont des étudiants de NCE III, dix-neuf (16) sont des étudiants de NCE II et vingt-sept (20) sont des étudiants de NCE I.

Tous nos sujets sont des yorouba maitrisant plus au moins le yorouba. Cette population couvre ces États: 24 viennent de l'État d'Ogun, 10 de l'État d'Oyo, 8 de l'État de Lagos, 4 de l'État d'Osun, 4 de l'État d'Ondo, 5 de l'État d'Ekiti et 3 de l'État de Kwara.

\subsection{Collecte des données}

L'objectif principal de cette étude est d'identifier les problèmes phonostylistiques de la lecture chez les apprenants yoroubaphones de français. Nous avons donné quatre tests différents aux étudiants à lire pour évaluer quatre éléments. Au niveau de l'exploitation des enregistrements, nous avons joué la cassette enregistrée trois fois pour écouter la lecture des tests donnés aux sujets enquêtés. À chaque écoute, nous prenons note des bonnes lectures aussi bien que des mauvaises lectures sur une feuille, l'analyse desquelles nous donnent les résultats obtenus.

\subsection{Analyse des Résultats \\ 4.2.1 L'accentuation}

L'accentuation est définie ici comme une inflexion prosodique particulière à une personne. C'est-à-dire le ton de la voix d'un homme grâce auquel l'on peut souligner ou mettre en valeur un énoncé.

Les sujets enquêtés ont des difficultés d'accentuation. On note l'absence d'accent d'intensité qui porte sur les dernières syllabes et qui est dit avec plus de force que les autres. Ce que nous entendons chez nos sujets est l'accent d'intensité sur la première syllabe de "inimaginable" dans la phrase (C'est inimaginable!), "difficile" dans la phrase (Philippe, la profession de comédien est difficile), "enfant" dans (Les enfants préparent leurs affaires). On note aussi l'accent d'insistance qui sert à modifier la hiérarchie des syllabes phonologiques. Ce qu'on remarque chez nos sujets est l'allongement du /a/ de "retardataire" dans (Je n'aime pas les retardataires), renforcement et élévation de ton de toute la syllabe. L'accent yoroubaphone se révèle aussi au niveau de la chute du /R/ final dans "pour" prononcé /pu/ au lieu de/puR/, "affaires" prononcé/afe/ au lieu de /afeR/.

\subsubsection{L'articulation}

L'articulation est la façon dont les organes articulatoires se réunissent pour contribuer à la réalisation d'une unité ou d'une suite d'unités. Autrement dit, ce sont les mouvements des organes phonatoires dans la formation des phonèmes.

Le test B est un passage. Dans ce test, on remarque des articulations fautives de "peut-être" articulé /postR/, au lieu de/pøt\&R/, "reviendrai-je" articulé/RovjedRe3o/ au lieu de /R $\square$ vjedRe3/. Dans le mot "un vieil

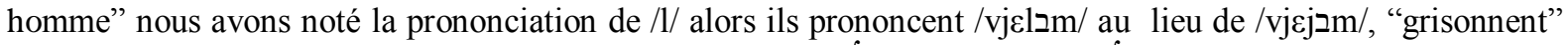
articulé /gRisonã/au lieu de/gRizבn/, "marcher" devient/maje/ au lieu de /maR/e/,

\subsubsection{L'intonation}

Dans la chaîne linéaire des sons du langage, il y a des variations de hauteur qui portent sur des éléments plus larges que les phonèmes ou les mots. Alors l'intonation est l'ensemble des variations de hauteur affectant l'énoncé oral.

Avant d'aborder l'aspect phonostylistique de l'intonation dans cette analyse, nous voulons mentionner que l'intonation au niveau linguistique est souvent confiné aux seuls cas où un énonce déclaratif est utilisé pour interroger, grâce à l'élévation de la voix. La phrase "Pourquoi Monique ?" en serait un bon exemple. Mais pour notre analyse, nous accordons de l'importance à la mélodie, à la ligne musicale de l'énoncé telle qu'elle est réalisée chez nos sujets, c'est-à-dire, la qualité montante et descendante de leur voix. 
Les schémas ci-dessous illustrent notre analyse où $\mathbf{A}$ représente la version standard et $\mathbf{B}$ représente la version peu standard produite par nos sujets.

A.

4.

3. À̀ cet age là

2. on n'est plus

1. un bébé

B.

4.

3.

2. À cet age là

1.

A.

4.

3. Mais il y a des parents

2.

1. qui aiment conserver leurs enfants

dans du coton

On remarque dans ces exemples que des sujets enquêtés ont des difficultés au niveau de l'intonation. On remarque que leur intonation ne descend pas comme il le faut, ce qui peut nous donner un autre sens du message du texte.

\subsubsection{La prononciation}

La prononciation est la manière dont les sons du langage sont articulés, c'est-à-dire la façon dont un mot est prononcé.

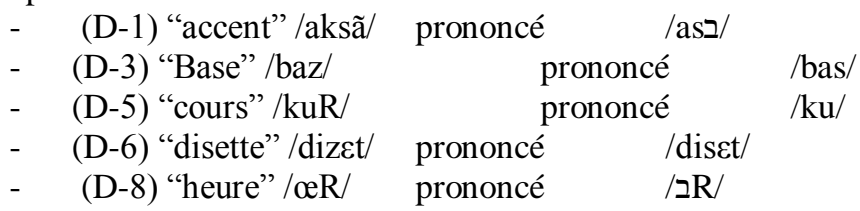

\section{Conclusion}

Dès le début de ce travail nous avons vu les différentes sortes de difficultés phonostylistiques que les étudiants nigérians apprenant le français peuvent rencontrer à travers la lecture en français. La plupart de ces difficultés sont attribuées aux phénomènes naturels; c'est-à-dire à l'interférence linguistique de la langue anglaise et des langues maternelles. Considérant les observations que nous avons faites chez nos enquêtés nous pouvons dire qu'un accent particulier soit mis sur l'enseignement de la lecture en français en général et chez les apprenants yoroubaphones du français.

D'autres difficultés que rencontrent les sujets enquêtés sont attribuées au professeur, au gouvernement et aux apprenants. Devant la désaffection croissante des apprenants pour la lecture, il est conseillé aux professeurs de français de prévoir un moment particulier pour l'enseignement de la lecture et ils doivent faire entrer les documents de toutes espèces et de tous niveaux dans la classe tels que: les journaux, les revues, les bandes dessinées, les textes publicitaires, etc. pour aider la capacité de lecture chez les apprenants. Mais comment peut-on enseigner ce qu'on maîtrise assez mal soi-même?

Puisque la plupart des professeurs de français nigérians ont appris le français dans le contexte plus ou moins semblable à celui de nos enquêtés, il devient impératif au gouvernement de s'assurer que tout professeur Nigérian de français aille en stage de formation dans un milieu naturellement francophone. Cette expérience pourrait par ailleurs être répétée afin de permettre à ces professeurs de maintenir une compétence communicative appréciable. La formation en linguistique africaine que nous proposons est de permettre aux professeurs de français d'avoir une connaissance du système phonique de ses apprenants afin de pouvoir ensuite faire des corrections phonétiques efficaces et rapides. D'autre part, la lecture en français dans les écoles pilotes doit figurer plusieurs fois par semaine dans l'horaire scolaire.

De la part du gouvernement, nous tenons à mentionner qu'il y a manque de matériels pédagogiques conçus localement, surtout pour les corrections phonétiques. Cela aussi contribue un autre handicap à la réussite de l'enseignement correcte de la lecture dans ce pays. Il faut des matériels tels que des magnétophones, des films français, des livres, un laboratoire et d'autres matériels indispensables à l'enseignement de la lecture. Le gouvernement peut organiser aussi des compétitions de lecture où les apprenants auront l'opportunité de présenter des lectures à l'oral.

D'après les enquêtes et les analyses que nous avons effectuées, nous constatons que les difficultés de la lecture qu'un étudiant nigérian du français rencontre à travers la langue française ne sont pas tout à fait sa faute. 
En fait, l'apprentissage du français ne commence qu'à partir de 11 ou 13 ans et cela correspond selon les psychologues tels que Pavlov, à la fin de la période la plus favorable à l'apprentissage d'une langue et pendant laquelle la faculté d'imitation de l'enfant est considérable. Par ceci, nous recommandons que l'apprentissage de la lecture en français commence de l'année primaire au lycée pour que les élèves puissent grandir avec le français.

Les apprenants doivent prendre l'habitude de lire pour améliorer leur orthographe, accroître leur vocabulaire et perfectionner leur style. Un apprenant qui a la passion pour les livres et la lecture peut arriver à la communication. La lecture paraît plus importante pour l'apprentissage d'une langue étrangère parce qu'elle permet d'acquérir l'attitude requise d'un bon lecteur. Très souvent, l'incapacité de lire et de comprendre les instructions engendre l'échec des candidats aux examens. Pour cette raison, on dit savoir lire, c'est savoir faire.

Finalement, avant qu'un étudiant ne puisse lire un texte d'une langue étrangère couramment, il faut qu'il ait le goût de lire diverses œuvres et des livres sur la bonne prononciation française. Il faut qu'il ait une radio avec laquelle il peut écouter les programmes français de la Radio France ou d'autres stations françaises pour acquérir une bonne prononciation.

\section{Theses:}

\section{References}

[1] Jimoh, Y. A. A. Phonologie Comparée du français et du yoruba: implications pour l'enseignement et l'apprentissage du français., Internet Thèse de doctorat non publiée, Département de Français, Faculté des Lettres, Université d'Ilorin, Nigéria, $2007,8$.

[2] Braille www.wikipédia.fr/lecture consulté le 20/10/09

\section{Article dans un magazine}

[3] Falcoz-Vigne «Texte Littéraire et activité de Lecture: Cadre Théorique» dans Le Français dans le Monde, Paris, no. 292 octobre

[4] 1997, 1987, p. 18

Livre

[5] Smith, F. Reading Without Nonsense. Portmouth: Heinemann, 1997.

[6] Bühler, K. Principes de phonologie. Paris: Klincksieck, 1934.

[7] Ladefoged, P. A Course in Phonetics. New York: Harcourt Brace Jovanovich, Inc. 1982

[8] Fonagy, I. La vive voix. Essais de psycho- phonétique. Paris: Payot, 1983.

\section{Artilce dans un journal}

[9] Ogunola, V.K. L'enseignement de la lecture dans l'apprentissage du français langue étrangère: Approche pédagogique" in Journal of Inter-College Association of French Teachers in Nigéria, Ibadan, Vol 5, No 1, 2003, pp. 214-229.

[10] Oloruntoba-Oju , Sociolinguistics: An Overview in Adegbija E. (ed.) The English Language and Literature in English. Ilorin, The Department of Modern European Languages, 1998, pp 146-158.

\section{Dictionaires}

[11] Encyclopédie Hachette, Paris, Hachette, 2003. Dictionnaire de Linguistique, Paris: Librairie Larousse, 1973 